Pacific

Journal of

Mathematics

COST-MINIMIZING NETWORKS

AMONG IMMISCIBLE FLUIDS IN $\mathbb{R}^{2}$

David Futer, Andrei Gnepp, David McMath, Brian A. Munson, Ting NG, Sang-Hyoun Pahk, and Cara Yoder 


\title{
COST-MINIMIZING NETWORKS AMONG IMMISCIBLE FLUIDS IN $\mathbb{R}^{2}$
}

\author{
David Futer, Andrei Gnepp, David Mcmath, Brian A. Munson, \\ Ting NG, Sang-Hyoun Pahk, and Cara Yoder
}

\begin{abstract}
We model interfaces between immiscible fluids as cost-minimizing networks, where "cost" is a weighted length. We consider conjectured necessary and sufficient conditions for when a planar cone is minimizing. In some cases we give a proof; in other cases we provide a counterexample.
\end{abstract}

\section{Introduction.}

In planar networks of soap films, segments meet in triples to form $120^{\circ}$ angles. Such angles are characteristic of nodes in length-minimizing networks. In this paper, we study the geometry of cost-minimizing planar networks, where we define cost to be weighted length. These networks have a much richer geometry than do soap-film networks. In particular, cost-minimizing networks can meet in any number around a node, with the angles between segments determined by their relative costs.

For examples of cost-minimizing networks, we look largely to immiscible fluids. When two immiscible fluids come together in a region of the plane, they meet to form an interface. This interface has an associated energy proportional to its length, which gives rise to a "cost constant." When several fluids come together, they form a network of interfaces, each interface with a specific cost constant. The fluids arrange themselves so as to minimize the total energy or cost. (Other generalizations of length-minimizing networks can be found in the surveys $[\mathbf{H R W}]$ and $[\mathbf{I T}]$.)

In general, one might impose area constraints on the regions occupied by the fluids. For this paper, though, we focus on the local behavior - how fluids come together at nodes. So we assume our networks are in the unit disk, and area constraints are replaced by constraints on how the regions meet the boundary circle. Our candidates for local minimizers are simply cones in the disk.

\section{The conjecture.}

We consider conjectured conditions for a cone to be minimizing. Conjecture 2.5 [FMMP, Conj. 2.7] states that a cone is minimizing if and only if a certain condition holds. This "calibration" condition involves placing a 
point for each fluid, with constraints on the vectors between points. Gary Lawlor and Frank Morgan proved that calibration is always sufficient for minimization and necessary if all fluids are present (Theorem 2.2). They leave open the question of whether the condition remains necessary if one allows additional fluids. For simple cases, we prove that it does (Theorem 4.4). In general, however, we prove by counterexample that it does not (Theorem 4.6). The primary tool that we use to prove the conjecture in simple cases is Theorem 2.9, in which we consider an uncalibrated cone and construct a cheaper competing network. To prove the counterexample, we combine a modified calibration argument with an estimate on competing networks obtained by filling regions with alternate fluids.

In the simplest case we consider, there is only one fluid absent from the minimizing cone. In this case, we show that if the cone were not calibrated we could insert a triangle of the extra fluid and decrease cost as in Figure 1.
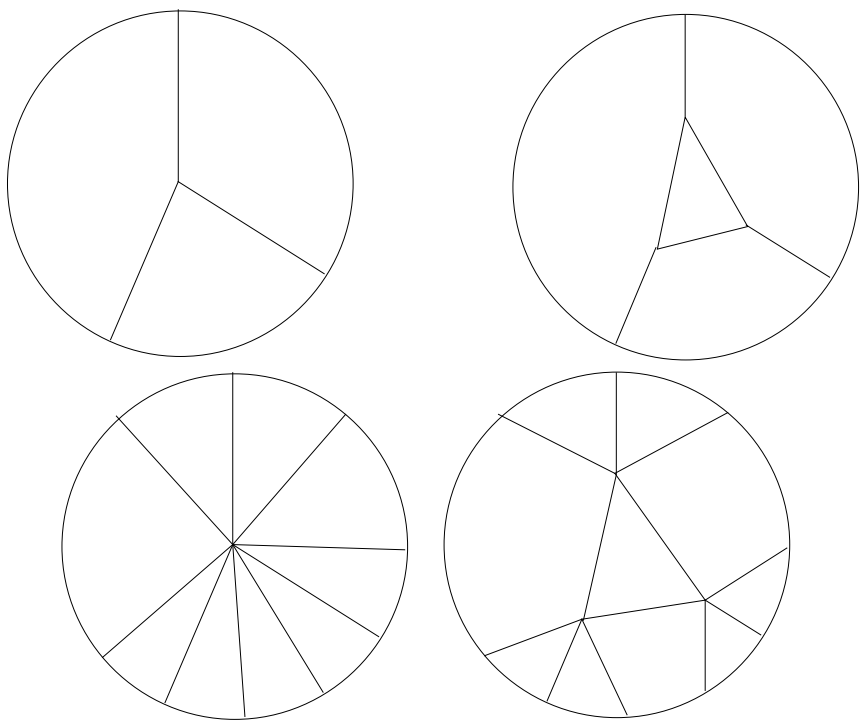

Figure 1. Inserting a triangular region of another fluid can reduce cost.

The case of two fluids absent introduces new complexities, and includes a counterexample. When the conjecture does hold, the competing network required by Theorem 2.9 takes the form of two triangles sharing an edge as in Figure 2. The counterexample arises when the two new fluids are only helpful in reducing cost by bordering diagonally opposite original fluids.

When more than two fluids are absent, the problem becomes considerably more complicated than the previous cases, even for just three fluids present and three absent [FMMP, $\S 7]$. In some subcases, it appears that we can 


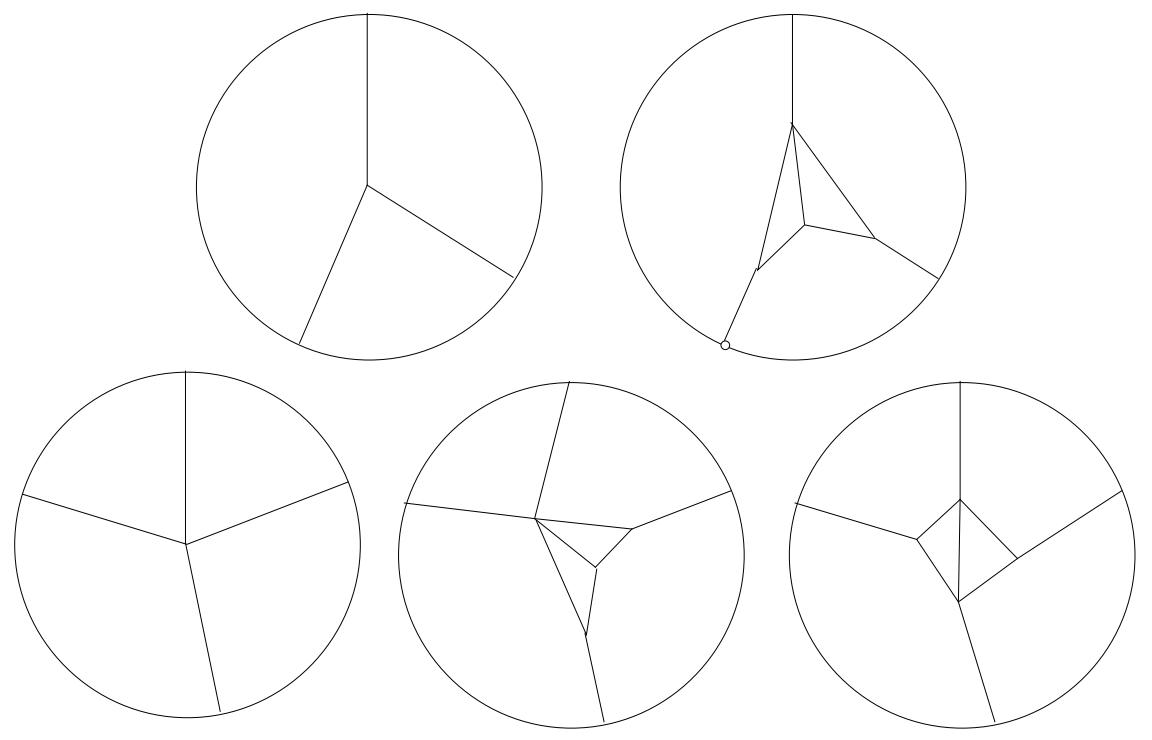

Figure 2. Inserting two triangular regions of other fluids can reduce cost.

prove that Conjecture 2.5 holds; in others, there will be counterexamples analogous to Theorem 4.6.

\section{The counterexample.}

A counterexample to Conjecture 2.5 must involve at least two absent and four present fluids (Theorem 4.4 and Remark 4.5). In fact, we use such a network; it is easy to show that the cone of our counterexample cannot be calibrated.

To show that our cone is minimizing, we need to show that adding the two absent fluids cannot reduce cost. We combine a modified calibration argument with calculations of the cost savings resulting from flooding competing networks with alternative fluids. The proof we give in this paper relies heavily on the flooding arguments to obtain contradictory estimates on interface lengths. An alternate proof involves a more elaborate calibration calculation, also taking into account the angles made by network boundaries [GNY, Thms. 1 and 2].

Our proof also helps to understand why a counterexample with two absent fluids requires at least four present fluids. The final contradiction we arrive at is that one extra fluid has to connect two diagonally opposite regions, and the other extra fluid has to connect the other diagonal pair. But this would require fluids to overlap, which is impossible.

\section{Previous work.}

The SMALL Geometry Groups 1995 [BTW] and 1996 [EMN] studied bubbles of immiscible fluids. Their work dealt with minimizing cost under area 


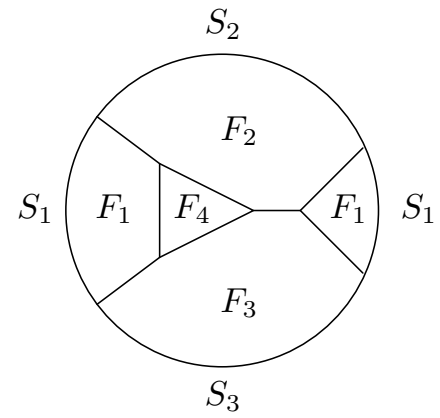

Figure 3. A network of six fluids, four of which are present $\left(R_{5}, R_{6}=\emptyset\right)$.

constraints. By focusing on local behavior instead of entire bubbles, we avoid some of the difficulties they encountered. Brian Elieson $[\mathbf{E}]$ obtained sufficient conditions for a bound on the number of fluids meeting at a point.

Acknowledgments. The authors were the members of the 1997 and 1998 Geometry Groups of the Williams College SMALL undergraduate research program, a National Science Foundation site for Research Experiences for Undergraduates, directed by Deborah Bergstrand and Thomas Garrity. Professor Frank Morgan advised both groups. We would like to thank Ken Brakke, Ronald L. Graham, Jon Kravis, Sam Payne, and Craig Westerland for their helpful conversations. The students at the SUNY Geneseo Workshop gave some motivation to Conjecture 2.5 by solving some symmetric cases. This work has been partially supported by National Science Foundation grants.

\section{Immiscible Fluids.}

Section 2 begins with definitions, known existence and regularity (Theorem 2.1), and calibration. Our Theorem 2.9 uses calibration to prove that certain perturbations of a given network have less cost.

\section{Definitions.}

We consider disjoint, relatively open regions $R_{1}, \ldots, R_{n}$, associated with fluids $F_{1}, \ldots, F_{n}$, in the closed unit disk $D$. These regions are separated by a network $N$ of $C^{1}$ curves meeting only at their endpoints at finitely many nodes, with $\bigcup R_{i}=D \backslash N$. (See Figure 3.)

Each region has a prescribed boundary arc $S_{i}=R_{i} \cap \partial D$, consisting of finitely many intervals. Fixed assignments of arcs $S_{i}$ to fluids $F_{i}$ are called boundary conditions. Each arc $S_{i}$ may be empty, in which case $R_{i}$ may or may not be empty. We will say that fluid $F_{i}$ is present or absent according as $R_{i}$ is nonempty or empty. In general, $R_{i}$ may have several 
components. For two regions $R_{i}$ and $R_{j}$ in a network $N$, we define the interface $N_{i j}=\partial R_{i} \cap \partial R_{j}$, oriented as the boundary of $R_{i}$. We may think of $N$ as either the union or the collection of interfaces $N_{i j}$, as dictated by context.

To every pair of fluids $F_{i}, F_{j}$ we assign a cost constant $a_{i j}=a_{j i}>0$. We seek networks that minimize an energy or cost

$$
\operatorname{cost}(N)=\sum_{i<j} a_{i j} \cdot \operatorname{length}\left(N_{i j}\right) .
$$

We assume that the cost constants for any fluids $F_{i}, F_{j}, F_{k}$ satisfy a triangle inequality

$$
a_{i k} \leq a_{i j}+a_{j k}
$$

(Otherwise, replacing $N_{i k}$ by an infinitesimal layer of $F_{j}$ would reduce cost.)

\section{Existence and regularity of cost-minimizing networks.}

Theorem 2.1 says that minimizing networks exist and consist of finitely many line segments.

Theorem 2.1 ([M2, Thm. 3.3 and Cor. 4.5]). Let $F_{1}, \ldots, F_{n}$ be a set of fluids with cost constants $a_{i j}$ that satisfy the strict triangle inequality $\left(a_{i k}<\right.$ $\left.a_{i j}+a_{j k}\right)$. For given boundary conditions, there exists a cost-minimizing network $N$. Furthermore, $N$ consists of finitely many line segments.

Frank Morgan [M2] and Brian White [W1], [W2] consider the more general problem with area constraints in the context of flat chains with coefficients in a group representing the fluids. Morgan uses geometric measure theory to prove that the minimizer must consist of constant-curvature arcs. In our problem, where we do not place area constraints on the fluids, the constant-curvature arcs are of course straight lines. Brian Elieson $[\mathbf{E}]$ attempts a direct proof of the existence of straight-line minimizing networks, although he succeeds only assuming a bound on the number of nodes.

\section{Calibration.}

Consider a network $N$, consisting of linear interfaces $N_{i j}$. For each $N_{i j} \neq \emptyset$, construct a unit normal vector $n_{i j} \perp N_{i j}$, pointing from $R_{i}$ into $R_{j}$. We say that points $p_{1}, \ldots, p_{n}$ calibrate $N$ if

$$
\begin{array}{ll}
p_{j}-p_{i}=a_{i j} n_{i j} & \text { when } N_{i j} \neq \emptyset, \text { and } \\
\left|p_{j}-p_{i}\right| \leq a_{i j} & \text { when } N_{i j}=\emptyset .
\end{array}
$$

From now on we focus our attention on the local behavior of a network in the neighborhood of a node, i.e., a cone, consisting of line segments meeting at the origin. Gary Lawlor and Frank Morgan $[\mathbf{L M}]$ prove that such networks minimize cost if and only if they are calibrated, assuming that all fluids are present. 
Theorem 2.2 ([LM, Thms. 2.1 and 2.5]). Let $N$ be a cone. If $N$ is calibrated, it is cost minimizing. Conversely, if $N$ is cost minimizing and each fluid is present, then $N$ can be calibrated.

Remark 2.3. If each fluid is present, then by (3) the points $p_{i}$ are unique, up to an additive constant.

Lemma 2.4. Consider a cone $N$ calibrated by points $p_{1}, \ldots, p_{n}$. Then the polygon $p_{1} \cdots p_{n}$ is strictly convex and each $R_{i}$ is connected.

Proof. Since $N$ is calibrated and hence minimizing, it follows easily that the angle between consecutive lines of $N$ is less than $180^{\circ}$. Since $p_{j}-p_{i}$ is normal to $N_{i j}$, it follows that $p_{1} \cdots p_{n}$ is strictly convex and that each $R_{i}$ is connected.

The conjecture we explore in this paper seeks to extend Theorem 2.2 and show that minimizing always implies calibrated.

Conjecture 2.5. A minimizing cone can be calibrated.

Remark 2.6. For one fluid present, the conjecture is trivially true. For two fluids present, the triangle inequality implies the conjecture [E, Prop. 6.1]. Thus, we will only consider cases with at least three fluids present.

Remark 2.7. Conjecture 2.5 holds for length-minimizing networks, when all cost constants $a_{i j}=1$. It is well known that for such networks, segments meet in triples to form $120^{\circ}$ angles ([HRW, §1.3, Thm. 1.1, p. 6], [IT, Ch. 3, Thm. 2.1, p. 120]). Such cones have an essentially unique calibration consisting of an equilateral triangle, and points for the absent fluids can be placed anywhere inside this triangle.

We will later show that this conjecture is false (Theorem 4.6), which leads us to the following open question.

Question 2.8. When can a minimizing cone be calibrated?

\section{Competing networks.}

The following theorem shows how to reduce the cost of certain networks by inserting absent fluids at a node. It will be used to prove in Theorems 3.1 and 4.4 that certain uncalibrated cones are not minimizing.

Theorem 2.9. Consider a smooth family $\{N(t)\}$ of networks of line segments $N_{i j}(t), 0 \leq t \leq T$ all satisfying the same boundary conditions, such that unless $i, j \leq m<n, N_{i j}(0)$ is empty and for all $0<t_{1}, t_{2} \leq T, N_{i j}\left(t_{2}\right)$ is parallel to $N_{i j}\left(t_{1}\right)$. Suppose $N(0)$ is calibrated by $p_{1}, \ldots, p_{m}$ for fluids $F_{1}, \ldots, F_{m}$, and suppose there are points $p_{m+1}, \ldots, p_{n}$ such that

- when $i, j \leq m$ and $N_{i j}(t) \neq \emptyset$, then $\left(p_{j}-p_{i}\right) \cdot n_{i j}(t)=a_{i j} \cos \theta_{i j}(t)$ (where $\theta_{i j}(t)$ is typically some small angle and $\theta_{i j}(0)=0$ because $N(0)$ is calibrated by $\left.p_{1}, \ldots, p_{n}\right)$; and 
- when $i>m$ or $j>m$ and $N_{i j}(t) \neq \emptyset$, then $p_{j}-p_{i}=\alpha_{i j} n_{i j}$, where $\alpha_{i j} \geq a_{i j}$ and $\alpha_{b c}>a_{b c}$ for some $N_{b c}(t)$ of length $t$. (The normal $n_{i j}$, and therefore $\alpha_{i j}$ as well, is independent of $t$, since $N_{i j}\left(t_{2}\right)$ is parallel to $N_{i j}\left(t_{1}\right)$.)

Then, for small positive $t, \operatorname{cost}(N(t))<\operatorname{cost}(N(0))$. In particular, $N(0)$ is not a minimizing network.

Proof. Let $\left|N_{i j}(t)\right|$ denote the length of $N_{i j}(t)$. Then

$$
\begin{aligned}
\operatorname{cost}(N(0)) & =\sum_{i<j \leq m} a_{i j}\left|N_{i j}(0)\right| \\
& =\sum_{i<j \leq m} \operatorname{flux}_{N_{i j}(0)}\left(p_{j}-p_{i}\right) \\
& =\sum_{i=1}^{m} \operatorname{flux}_{S_{i}}\left(p_{i}\right) \\
& =\sum_{i=1}^{n} \operatorname{flux}_{S_{i}}\left(p_{i}\right) \\
& =\sum_{i<j \leq n} \operatorname{flux}_{N_{i j}(t)}\left(p_{j}-p_{i}\right),
\end{aligned}
$$

and

$$
\begin{aligned}
\operatorname{cost}(N(t)) & =\sum_{i<j \leq n} a_{i j}\left|N_{i j}(t)\right| \\
& =\sum_{i<j \leq m} a_{i j}\left|N_{i j}(t)\right|+\sum_{\substack{i<j \\
m<j}} a_{i j}\left|N_{i j}(t)\right| .
\end{aligned}
$$

Combining these two equations, we get

$$
\begin{aligned}
\operatorname{cost}(N(t))-\operatorname{cost}(N(0))= & \sum_{\substack{i<j \leq m\\
}}\left(a_{i j}\left|N_{i j}(t)\right|-\operatorname{flux}_{N_{i j}(t)}\left(p_{j}-p_{i}\right)\right) \\
& +\sum_{\substack{i<j \\
m<j}}\left(a_{i j}\left|N_{i j}(t)\right|-\operatorname{flux}_{N_{i j}(t)}\left(p_{j}-p_{i}\right)\right) \\
= & \sum_{\substack{i<j \leq m \\
\text { i }}} a_{i j}\left(1-\cos \theta_{i j}\right)\left|N_{i j}(t)\right| \\
& +\sum_{\substack{i<j \\
m<j}}\left(a_{i j}-\alpha_{i j}\right)\left|N_{i j}(t)\right| .
\end{aligned}
$$

Now we can calculate the derivative of $\operatorname{cost}(N(t))$ at $t=0$. Here, we must recall that $\theta_{i j}(0)=0$. Recall also that $\left|N_{i j}(0)\right|=0$ if $j>m$. Since 
$\left|N_{i j}\right|^{\prime}(0) \geq 0$ for all $j>m$, therefore every $\left(a_{i j}-\alpha_{i j}\right)\left|N_{i j}\right|^{\prime}(0) \leq 0$. Furthermore, since some $\alpha_{b c}>a_{b c}$, this $\left(a_{b c}-\alpha_{b c}\right)\left|N_{b c}\right|^{\prime}(0)<0$. Hence

$$
\begin{aligned}
& \left.\frac{d}{d t}\right|_{t=0} \operatorname{cost}(N(t)) \\
& =\left.\frac{d}{d t}\right|_{t=0} \operatorname{cost}(N(t))-\operatorname{cost}(N(0)) \\
& =\sum_{i<j \leq m} a_{i j}\left(\sin \theta_{i j}(0) \theta_{i j}^{\prime}(0)\left|N_{i j}(0)\right|\right. \\
& \left.\quad+\left(1-\cos \theta_{i j}(0)\right)\left|N_{i j}\right|^{\prime}(0)\right)+\sum_{\substack{i<j \\
m<j}}\left(a_{i j}-\alpha_{i j}\right)\left|N_{i j}\right|^{\prime}(0) \\
& =\sum_{\substack{i<j \\
m<j}}\left(a_{i j}-\alpha_{i j}\right)\left|N_{i j}\right|^{\prime}(0) \\
& <0 .
\end{aligned}
$$

Therefore, for sufficiently small $t>0, N(t)$ is a cheaper network than $N(0)$.

\section{One fluid absent.}

Theorem 3.1 considers a minimizing network $N$ with one fluid absent say $F_{n}$ - and applies Theorem 2.9 to prove that $N$ can be calibrated. By Theorem 2.2, we already know there is a calibration $p_{1}, \ldots, p_{n-1}$ for fluids $F_{1}, \ldots, F_{n-1}$. To place $p_{n}$ within $a_{i n}$ of $p_{i}$ for $i \leq n-1$, we just need to know that the intersection of the disks $D_{i}=\mathrm{D}\left(p_{i}, a_{i n}\right)$ is nonempty (see Figure 4).

Theorem 3.1. Let $N$ be a minimizing cone. If at most one fluid is absent, then $N$ can be calibrated.

Proof. If no $R_{i}$ is empty, the result reduces to Theorem 2.2. Thus we may suppose without loss of generality that $R_{n}$ is empty. We will show that if $N$ cannot be calibrated, then it must not be a minimizer, by constructing a cheaper network with a nonempty $R_{n}$.

Suppose $N$ cannot be calibrated. By definition, the intersection of the disks $D_{i}=\mathrm{D}\left(p_{i}, a_{i n}\right)$ is empty. Then, by Helly's Theorem [Lay, Thm. 6.2], there exist $1<b<c \leq n-1$ (possibly after relabeling) such that $D_{1} \cap D_{b} \cap D_{c}=\emptyset$. Place $p_{n}$ in the region omitted by the three disks; note that $p_{n} \in \triangle p_{1} p_{b} p_{c}$.

For small $t>0$, construct a triangle

$$
T(t)=\left\{N_{1 n}(t), N_{b n}(t), N_{c n}(t)\right\},
$$




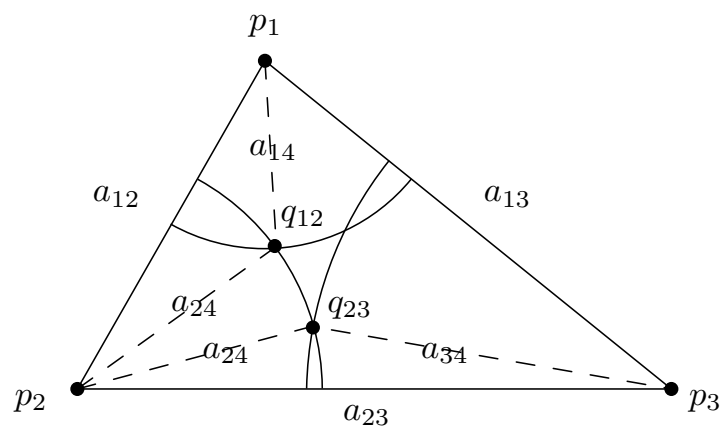

Figure 4. The disks $\mathrm{D}\left(p_{i}, a_{i 4}\right)$ must intersect pairwise because of the triangle inequality. However if all three have no common intersection, point $p_{4}$ cannot be placed close enough to the others to satisfy (4). So the corresponding three-fluid network will be minimizing if and only if this common intersection of the three disks $\mathrm{D}\left(p_{i}, a_{i 4}\right)$ is nonempty; this happens if and only if $\angle p_{3} p_{2} q_{23}+\angle p_{1} p_{2} q_{12} \geq \angle p_{1} p_{2} p_{3}$.

where these $N_{i n}(t)$ are normal to the segments $\overline{p_{i} p_{n}}$ as in Figure 5 and $t=\left|N_{1 n}(t)\right|$. Since $p_{n} \in \triangle p_{1} p_{b} p_{c}, \partial T(t)$ consists of, in counterclockwise order, $N_{1 n}(t), N_{b n}(t)$, and $N_{c n}(t)$. Translate $T(t)$ so that its center of mass lies at the origin; then $T(t)$ shrinks to the origin as $t \rightarrow 0$.

Now add segments $N_{i(i+1)}(t)$ connecting $N_{i(i+1)} \cap \partial D$ (the prescribed boundary) to the appropriate vertices of $T(t)$. For $1 \leq i<b$, this will be $N_{1 n}(t) \cap N_{b n}(t)$; for $b \leq i<c$, this will be $N_{b n}(t) \cap N_{c n}(t)$; and for $c \leq i \leq n-1$, this will be $N_{c n}(t) \cap N_{1 n}(t)$, as in Figure 5 .

Then we have a family of networks $N(t)=T(t) \cup\left\{N_{i(i+1)}(t)\right\}$, with fluid $F_{n}$ filling the interior of $T(t)$. It follows by Theorem 2.9 that $N=N(0)$ is not minimizing.

\section{An algebraic criterion for one of four fluids absent.}

Theorems 2.2 and 3.1 (with $n=3$ ) establish necessary and sufficient conditions for whether a calibrated cone $N$ separating three fluids can be improved by adding a fourth fluid. Theorem 3.2 reduces those conditions to an algebraic inequality on the cost constants $a_{i j}$.

Theorem 3.2. Consider a minimizing network $N$ consisting of three line segments meeting at a node, separating fluids $F_{1}, F_{2}, F_{3}$. Its cost can be reduced by adding a fourth fluid if and only if

$$
\begin{aligned}
\cos ^{-1}\left(\frac{a_{23}^{2}+a_{24}^{2}-a_{34}^{2}}{2 a_{23} a_{24}}\right)+\cos ^{-1}\left(\frac{a_{12}^{2}+a_{24}^{2}-a_{14}^{2}}{2 a_{12} a_{24}}\right) \\
<\cos ^{-1}\left(\frac{a_{12}^{2}+a_{23}^{2}-a_{13}^{2}}{2 a_{12} a_{23}}\right) .
\end{aligned}
$$



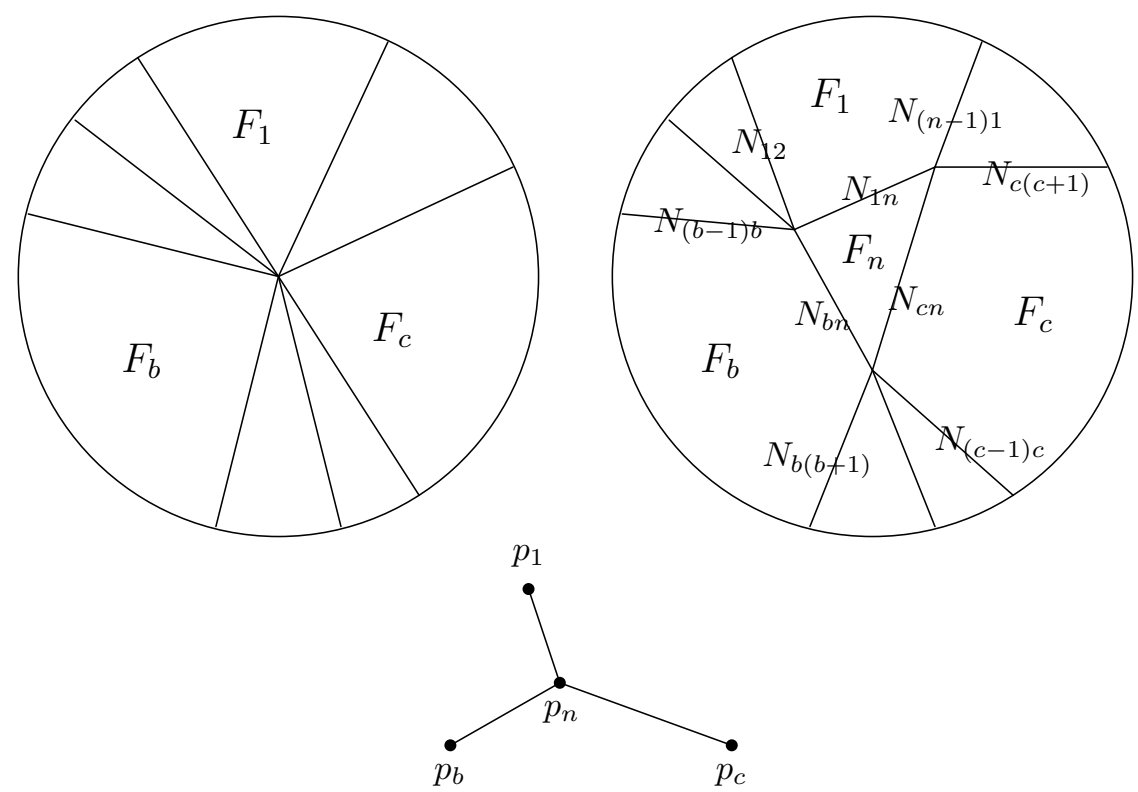

Figure 5. Starting with an uncalibrated network (left), we know that $\triangle p_{1} p_{b} p_{c}$ contains $p_{n}$ (bottom). Hence we can construct a comparison network (right) containing the triangle $T(t)=\left\{N_{1 n}(t), N_{b n}(t), N_{c n}(t)\right\} . \quad\left(N_{i j}(t)\right.$ separates fluids $F_{i}$ and $F_{j}$; for simplicity we have dropped the " $(t)$ "s in the diagram.) This comparison network beats the original network.

Proof. In light of Theorems 2.2 and 3.1, it suffices to show that inequality (5) holds if and only if the essentially unique calibration $p_{1}, p_{2}, p_{3}$ for fluids $F_{1}, F_{2}, F_{3}$ cannot be extended to a calibration $p_{1}, \ldots, p_{4}$ for fluids $F_{1}, \ldots, F_{4}$, i.e., if and only if the intersection $E$ of the three disks $\mathrm{D}\left(p_{i}, a_{i 4}\right)$ is empty.

Letting $q_{i j}=\partial \mathrm{D}\left(p_{i}, a_{i 4}\right) \cap \partial \mathrm{D}\left(p_{j}, a_{j 4}\right)$ (see Figure 4), we see that this occurs if and only if

$$
\angle p_{3} p_{2} q_{23}+\angle p_{1} p_{2} q_{12}<\angle p_{1} p_{2} p_{3} .
$$

By the law of cosines, this reduces to (5).

Remark 3.3. In the symmetric case where $a_{12}=a_{13}=a_{23}=1$ and $a_{14}=$ $a_{24}=a_{34}=a$, the inequality (5) reduces to

$$
2 \cos ^{-1}\left(\frac{1}{2 a}\right)<\cos ^{-1}\left(\frac{1}{2}\right)=\frac{\pi}{3} \text {. }
$$

This holds only when $\cos ^{-1}\left(\frac{1}{2 a}\right)<\frac{\pi}{6}$, or $a<\frac{1}{\sqrt{3}}$. Students at the SUNY Geneseo Student Workshop in April 1997 [M1] proved this case. 
The Geneseo students also considered a symmetric case of adding a fifth fluid to a minimizing cone separating $F_{1}, F_{2}, F_{3}, F_{4}$. Here, $a_{12}=a_{23}=$ $a_{34}=a_{34}=1, a_{13}=a_{24}=\sqrt{2}$, and $a_{i 5}=a$. In this case, the original network can be made cheaper by adding the fifth fluid if $a<\frac{1}{\sqrt{2}}$, and (3) and (4) can be satisfied if $a \geq \frac{1}{\sqrt{2}}$. Extending the results of this section to that case would be complicated.

\section{Two fluids absent.}

Section 4 considers a minimizing cone $N$ of $n$ fluids, with two fluids absent, say $F_{n-1}$ and $F_{n}$. Theorem 4.4 proves that $N$ can be calibrated, with one possible exception. By Remark 2.6 we can assume $n \geq 5$; the exception first occurs when $n=6$ (four fluids present, two absent). Theorem 4.6 will later show that the exceptional case yields a counterexample. Before showing that minimizing implies calibrated (with the stated exception), we must first introduce a few concepts.

\section{"Eyes".}

An eye is the intersection of (closed) disks. An arc of the eye is a circular arc making up part of the boundary of the eye. A vertex of the eye is an intersection of two arcs.

Define the two eyes

$$
E_{n-1}=\bigcap_{i=1}^{n-2} D\left(p_{i}, a_{i(n-1)}\right) \quad \text { and } \quad E_{n}=\bigcap_{i=1}^{n-2} D\left(p_{i}, a_{i n}\right) .
$$

We will call $p_{i}$ a cost-realizing point for $p_{n} \in E_{n}$ if $\left|p_{i}-p_{n}\right|=a_{i n}$.

We wish to show that (with the stated exception) any minimizing network can be calibrated. To do this, we will show that any network that cannot be calibrated can be beaten. By Theorem 2.2, any network without a calibration for $F_{1}, \ldots, F_{n-2}$ can be beaten (using only fluids $F_{1}, \ldots, F_{n-2}$ ). By Theorem 3.1, any network with $E_{n-1}=\emptyset$ or $E_{n}=\emptyset$ can be beaten (using only $n-1$ fluids). Therefore, for the purposes of the following lemmas, we assume that

- $p_{1}, \ldots, p_{n-2}$ calibrate $F_{1}, \ldots, F_{n-2}$; and

- $E_{n-1}$ and $E_{n}$ are nonempty.

Let us place $p_{n-1} \in E_{n-1}$ and $p_{n} \in E_{n}$ so as to minimize $\left|p_{n-1}-p_{n}\right|$. If there is no calibration of $N$ for all $n$ fluids $F_{1}, \ldots, F_{n}$, then

$$
\left|p_{n-1}-p_{n}\right|>a_{(n-1) n} .
$$

Lemma 4.1. The points $p_{n-1} \in E_{n-1}$ and $p_{n} \in E_{n}$ which minimize $\left|p_{n-1}-p_{n}\right|$ lie on vertices of $E_{n-1}$ and $E_{n}$.

Proof. Let $\alpha=\left|p_{n-1}-p_{n}\right|$. Clearly $\mathrm{D}\left(p_{n-1}, \alpha\right)$ and $E_{n}$ meet only at a single point, which is $p_{n}$. If $p_{n}$ is on the interior of the $p_{i}$-arc of the eye $E_{n}$, then 
the two circles $\operatorname{circ}\left(p_{n-1}, \alpha\right)$ and $\operatorname{circ}\left(p_{i}, a_{i n}\right)$ are tangent. It follows that $p_{n} \in \overline{p_{i} p_{n-1}}$. By inequality (7), $\alpha>a_{(n-1) n}$ and

$$
\begin{aligned}
a_{i(n-1)} & \geq\left|p_{i}-p_{n-1}\right| \\
& =\left|p_{i}-p_{n}\right|+\left|p_{n}-p_{n-1}\right| \\
& =a_{i n}+\alpha \\
& >a_{i n}+a_{(n-1) n},
\end{aligned}
$$

contradicting the triangle inequality for cost constants. So $p_{n}$ cannot be in the interior of an arc on the eye, and it must be at a vertex. Similarly, $p_{n-1}$ must be on a vertex of $E_{n-1}$.

Lemma 4.2. Suppose that $p_{n-1} \in E_{n-1}$ and $p_{n} \in E_{n}$ are placed as close together as possible. Then there exist two cost-realizing points $p_{i}$ and $p_{j}$ for $p_{n-1}$ such that $p_{n-1} \in \triangle p_{i} p_{j} p_{n}$.

Proof. Consider the convex hull of $p_{n}$ along with all of the cost-realizing points for $p_{n-1}$. (By Lemma 4.1 there are at least two cost-realizing points for $p_{n-1}$.) If $p_{n-1}$ were not inside this convex hull, moving it closer to the nearest point of the convex hull would decrease the distance between $p_{n-1}$ and all of the points involved in the convex hull (i.e., $p_{n}$ and the cost-realizing points). Since we chose $p_{n-1} \in E_{n-1}$ to be as close to $p_{n}$ as possible, this cannot happen. Hence $p_{n-1}$ lies inside the convex hull of $p_{n}$ and the costrealizing points. Since this is just the union of triangles formed by $p_{n}$ and pairs of cost-realizing points, there is a pair $\left\{p_{i}, p_{j}\right\}$ of cost-realizing points such that $p_{n-1} \in \triangle p_{n} p_{i} p_{j}$.

We will say that $p_{n-1}$ lies on the $p_{i}-p_{j}$ vertex of its eye $E_{n-1}$ if $p_{n-1} \in E_{n-1}$ and $p_{n} \in E_{n}$ are placed as closed together as possible and $p_{i}$ and $p_{j}$ satisfy the conclusion of Lemma 4.2, i.e., if $p_{i}$ and $p_{j}$ are cost-realizing points for $p_{n-1}$ such that $p_{n-1} \in \triangle p_{i} p_{j} p_{n}$.

Lemma 4.3. Suppose $p_{n-1} \in E_{n-1}$ and $p_{n} \in E_{n}$ have been chosen to minimize $\left|p_{n-1}-p_{n}\right|$. Then $p_{n-1}$ and $p_{n}$ cannot both be on $p_{b}-p_{c}$ vertices of their eyes.

Proof. If both $p_{n-1}$ and $p_{n}$ are on $p_{b}-p_{c}$ vertices of $E_{n-1}$ and $E_{n}$, then by Lemma 4.2, $p_{n-1} \in \triangle p_{b} p_{c} p_{n}$ and $p_{n} \in \triangle p_{b} p_{c} p_{n-1}$. But this can hold only if $p_{b}, p_{c}, p_{n-1}, p_{n}$ are collinear, which cannot happen by triangle inequality considerations similar to those in Lemma 4.1. It follows that $p_{n-1}$ and $p_{n}$ cannot both be on $p_{b}-p_{c}$ vertices.

\section{Two fluids absent.}

We can now state and prove our theorem about two fluids absent. It is here that we encounter a counterexample to Conjecture 2.5. We do, however, prove that many types of minimizing networks can be calibrated. Theorem 4.6 provides a counterexample to Conjecture 2.5 at the end of the section. 
Suppose that $p_{n-1} \in E_{n-1}$ and $p_{n} \in E_{n}$ are placed as close together as possible, with $p_{n-1}$ on the $p_{b}-p_{c}$ vertex of $E_{n-1}$ and $p_{n}$ on the $p_{d}-p_{e}$ vertex of $E_{n}$. Let us renumber $p_{1}, \ldots, p_{n-2}$ in a counterclockwise order, so that (without loss of generality) $b<c$ and $d<e$. We will say that $p_{n-1}$ and $p_{n}$ are on opposite vertices of their eyes if $b<d<c<e$.

Theorem 4.4. Consider a minimizing cone $N$. If at most two regions $R_{i}$ are empty, then $N$ can be calibrated, with the following possible exception: placing the points - say, $p_{n-1}$ and $p_{n}$ - for two absent fluids as close as possible while still close enough to the essentially uniquely placed other $p_{i}$ puts them on opposite vertices of the eyes $E_{n-1}$ and $E_{n}$.

Remark 4.5. When $n=5$, there are no opposite vertices for $p_{n-1}$ and $p_{n}$ to lie on since all fluids in the original network are adjacent. However, when $n \geq 6$, this situation can occur, and Theorem 4.6 provides a counterexample to Conjecture 2.5.

Proof. If at most one $R_{i}$ is empty, the result reduces to Theorem 3.1. Thus we may suppose without loss of generality that regions $R_{n-1}$ and $R_{n}$ are empty. We proceed by contradiction, supposing that $N$ cannot be calibrated and that the exceptional condition does not hold.

Recall that $p_{1}, \ldots, p_{n-2}$ are the essentially unique calibration for fluids $F_{1}, \ldots, F_{n-2}$ and that $p_{n-1} \in E_{n-1}$ and $p_{n} \in E_{n}$ have been chosen to minimize the distance between them. To derive a contradiction, we will assume that inequality (7) holds and then produce a family $\{N(t)\}$ of competing networks of less cost.

By Lemma $4.2, p_{n-1}$ lies on a $p_{b}-p_{c}$ vertex and $p_{n}$ lies on a $p_{d}-p_{e}$ vertex. Relabel if necessary so that $p_{1}, \ldots, p_{n-2}$ are in counterclockwise order (so the cone $N$ consists of interfaces $N_{i(i+1)}$ with indices taken modulo $n-2$ ) with $1=b<c$ and $d<e$. By Lemma 4.3, we may take $b<d$ as well. We do not consider $b<d<c<e$, so we have only the cases where $b<c \leq d<e$.

Now, we know that the polygon $p_{1} \cdots p_{n-2}$ is convex by Lemma 2.4. Also, $p_{n-1} \in \triangle p_{1} p_{c} p_{n}$ and $p_{n} \in \triangle p_{d} p_{e} p_{n-1}$ by Lemma 4.2 . Hence the four polygons at the top of Figure 6 ,

$$
\begin{aligned}
P_{1} & =p_{1} p_{2} \cdots p_{c-1} p_{c} p_{n-1}, \\
P_{2} & =p_{c} p_{c+1} \cdots p_{d-1} p_{d} p_{n} p_{n-1}, \\
P_{3} & =p_{d} p_{d+1} \cdots p_{e-1} p_{e} p_{n}, \text { and } \\
P_{4} & =p_{e} p_{e+1} \cdots p_{n-2} p_{1} p_{n-1} p_{n},
\end{aligned}
$$

are all convex, regardless of whether $c=d$ (in which case $P_{2}$ is just a triangle) or $c<d$.

For $t>0$ small, construct a split quadrilateral

$$
Q(t)=\left\{N_{1(n-1)}(t), N_{c(n-1)}(t), N_{(n-1) n}(t)\right\} \cup\left\{N_{(n-1) n}(t), N_{d n}(t), N_{e n}(t)\right\}
$$



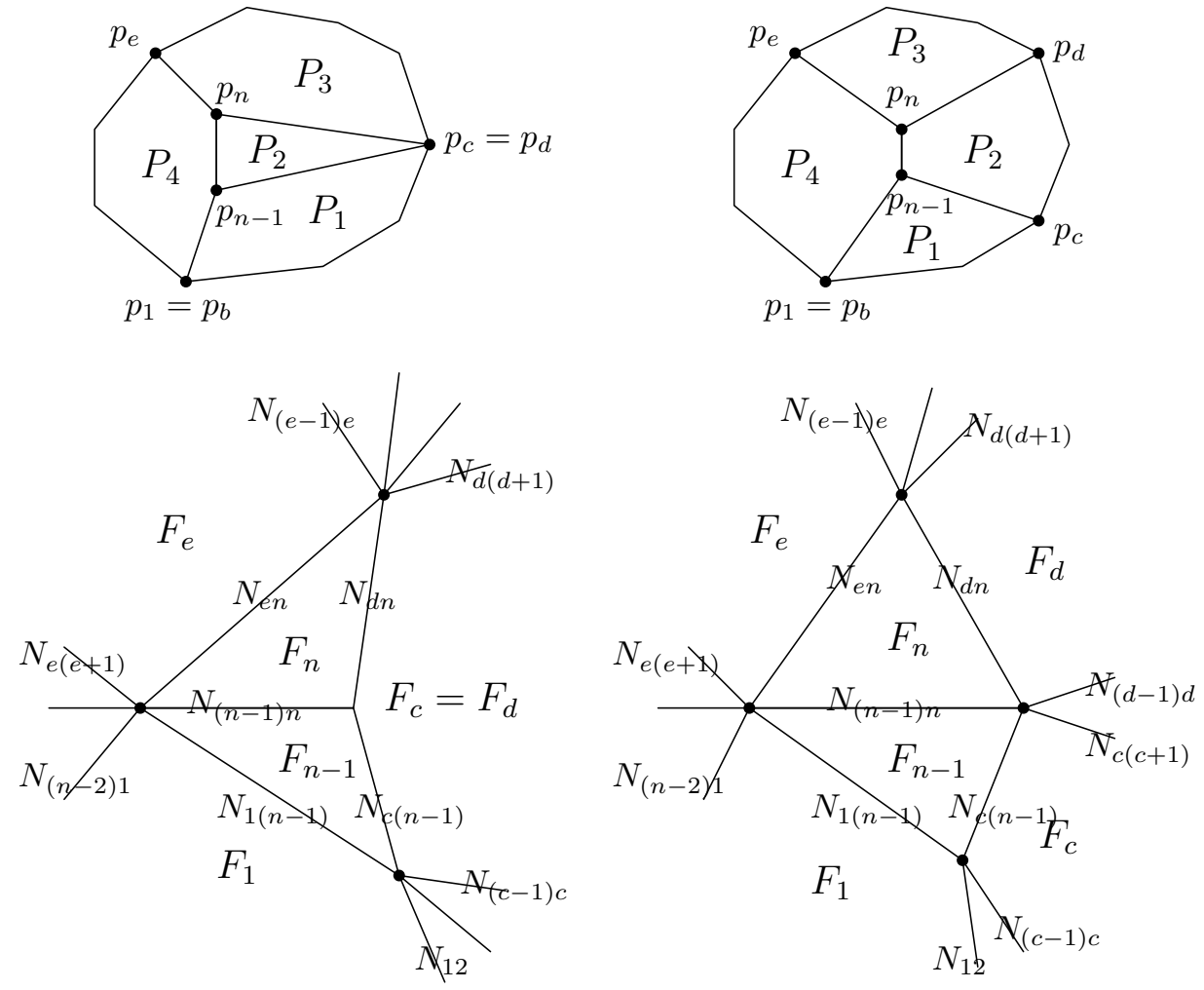

Figure 6. Since the polygons $P_{1}, P_{2}, P_{3}, P_{4}$ (top) are convex, we can construct a comparison network containing the quadrilateral $Q(t)$ (bottom). ( $N_{i j}(t)$ separates fluids $F_{i}$ and $F_{j}$; for simplicity we have dropped the " $(t)$ "s in the diagram.) This works regardless of whether $c=d$ (left) or $c<d$ (right).

composed of two triangles sharing an edge, with these $N_{i j}(t)$ normal to the segments $\overline{p_{i} p_{j}}$ (see Figure 6, bottom) and $t=\left|N_{(n-1) n}(t)\right|$. The convexity of polygons $P_{i}$ ensures that the two triangles

$$
\begin{aligned}
\triangle_{n-1}(t) & =\left\{N_{1(n-1)}(t), N_{c(n-1)}(t), N_{(n-1) n}(t)\right\} \\
\triangle_{n}(t) & =\left\{N_{(n-1) n}(t), N_{d n}(t), N_{e n}(t)\right\}
\end{aligned}
$$

are disjoint except for their shared edge $N_{(n-1) n}(t)$; and also ensures that $\partial Q(t)$ consists of, in counterclockwise order, the edges $N_{1(n-1)}(t), N_{c(n-1)}(t)$, $N_{d n}(t), N_{e n}(t)$. Furthermore, translate $Q(t)$ so that its center of mass lies at the origin; then, as $t \rightarrow 0, Q(t)$ shrinks to the origin.

Now, add segments $N_{i(i+1)}(t)$ connecting $N_{i(i+1)} \cap \partial D$ (the prescribed boundary) to the appropriate vertices of $Q(t)$. For $1 \leq i<c$, this will 


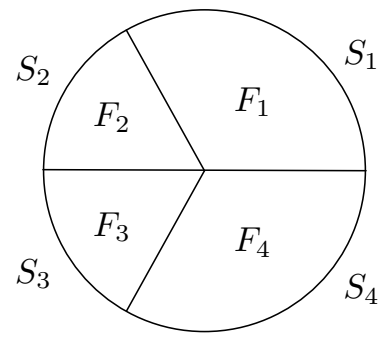

Figure 7. A counterexample to Conjecture 2.5, consisting of four present fluids and two absent. The network is minimizing but cannot be calibrated.

be $N_{1(n-1)}(t) \cap N_{c(n-1)}(t)$; for $c \leq i<d$ (if such $i$ exist), this will be $N_{c(n-1)}(t) \cap N_{d n}(t)$; for $d \leq i<e$, this will be $N_{d n}(t) \cap N_{e n}(t)$; and for $e \leq i \leq n-2$, this will be $N_{e n}(t) \cap N_{1(n-1)}(t)$. (See Figure 6, bottom.)

Then we have a family of networks $N(t)=Q(t) \cup\left\{N_{i(i+1)}(t)\right\}$, with fluid $F_{n-1}$ filling the interior of $\triangle_{n-1}(t)$ and fluid $F_{n}$ in the interior of $\triangle_{n}(t)$. It follows by Theorem 2.9 that $N=N(0)$ is not minimizing.

\section{A counterexample to the conjecture.}

Finally, we prove a counterexample to Conjecture 2.5.

Theorem 4.6. Consider the network $N$ consisting of four straight lines meeting at a node separating a disc into four regions of fluids $F_{1}, F_{2}, F_{3}$, $F_{4}$ as in Figure 7 . There exist cost constants $a_{i j}(1 \leq i, j \leq 6)$ satisfying the strict triangle inequality such that $N$ cannot be calibrated, but is still minimizing.

Proof. We define six points $p_{1}, p_{2}, \ldots, p_{6}$ in $\mathbb{R}^{2}$ as in Figure 8:

$$
\begin{aligned}
& p_{1}=\left(\cos 30^{\circ}, 2\right) \\
& p_{2}=(0,1.5) \\
& p_{3}=(0,0.5) \\
& p_{4}=\left(\cos 30^{\circ}, 0\right) \\
& p_{5}=\left(\cos 30^{\circ}-0.11,0.94\right) \\
& p_{6}=\left(\cos 30^{\circ}-0.11,1.06\right) .
\end{aligned}
$$

The cost constants are given by $a_{i j}=\left|p_{i}-p_{j}\right|+g(i, j) \epsilon$, where $\epsilon$ is a small positive constant chosen to be $10^{-9}$ and $g(i, j)$ is defined as follows:

$$
g(i, j)= \begin{cases}60000 & \text { if }(i, j)=(1,5),(3,5),(2,6) \text { or }(4,6) \\ -1 & \text { if }(i, j)=(5,6) \\ 0 & \text { otherwise }\end{cases}
$$




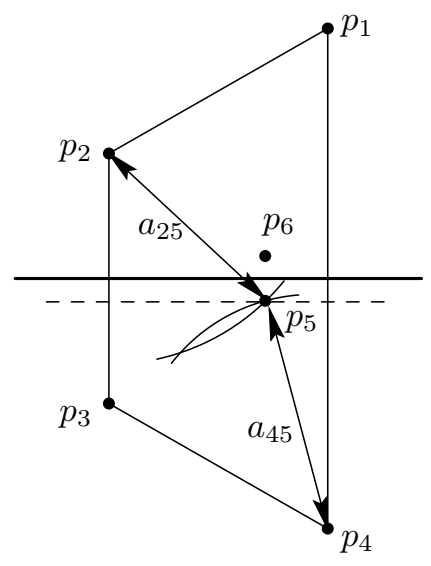

Figure 8. The points $p_{1}, p_{2}, p_{3}, p_{4}$ calibrate the network for four fluids, but an attempted placement of a point $q_{5}$ for fluid $F_{5}$ must lie below the dotted line. The heavy line is the axis of symmetry.

The numerical values of the cost constants are given below.

$$
\begin{gathered}
a_{12}=a_{23}=a_{34}=1 \\
a_{13}=a_{24}=\sqrt{3} \approx 1.73205080757 \\
a_{14}=2 \\
a_{15}=a_{46} \approx 1.06575226327 \\
a_{16}=a_{45} \approx 0.94641428561 \\
a_{25}=a_{36} \approx 0.94083708003 \\
a_{26}=a_{35} \approx 0.87480248277 \\
a_{56}=0.119999999 .
\end{gathered}
$$

It is easy to check by hand that strict triangle inequalities are satisfied. Alternatively, observe that the cost constants are equal to the Euclidean distances between corresponding points, sometimes with small corrections. The triangle inequality for the Euclidean metric thus implies strict triangle inequality for our cost constants with $\epsilon$ small, since no three points $p_{i}, p_{j}, p_{k}$ lie on the same line.

Now, we claim that no calibration for $F_{1}, F_{2}, \ldots, F_{6}$ exists for network $N$. For suppose that $\left\{q_{1}, q_{2}, \ldots, q_{6}\right\}$ calibrated $N$ with respect to $F_{1}, F_{2}, \ldots, F_{6}$. Since $a_{i j}=\left|p_{i}-p_{j}\right|$ for $1 \leq i<j \leq 4$, the placing of points $q_{1}, q_{2}, q_{3}$ and $q_{4}$ is essentially unique. So we may assume $q_{1}, q_{2}, q_{3}, q_{4}$ coincide with $p_{1}, p_{2}, p_{3}, p_{4}$, respectively, as in Figure 8. Since $a_{25}=\left|p_{2}-p_{5}\right|$ and $a_{45}=\left|p_{4}-p_{5}\right|$, we conclude that $q_{5}$ lies (horizontally) below $p_{5}$. Similarly, $q_{6}$ must lie above $p_{6}$. 
Since $p_{5}$ and $p_{6}$ lie on the same vertical line, $\left|q_{5}-q_{6}\right| \geq\left|p_{5}-p_{6}\right|=a_{56}+\epsilon>$ $a_{56}$, and it follows that $N$ cannot be calibrated.

The difficult part is to show that $N$ is cost-minimizing for $F_{1}, F_{2}, \ldots, F_{6}$. Suppose not; let $M$ be a minimizing network. Since by Theorem $2.2 N$ is minimizing for fluids $\left\{F_{1}, F_{2}, F_{3}, F_{4}, F_{5}\right\}$ and for $\left\{F_{1}, F_{2}, F_{3}, F_{4}, F_{6}\right\}$, both $F_{5}$ and $F_{6}$ must be present in the network $M$.

In the network $M$ with interfaces $M_{i j}$, let $R_{i}$ denote the region of fluid $F_{i}$.

The main technique of our proof will be a process called flooding. We consider the alternative network formed when we flood a particular region of $M$ with a different fluid. Since $M$ is already cost-minimizing, this process cannot reduce cost. We will use this fact to generate bounds on the lengths of various interfaces.

The next step of our proof is to show that the region $R_{1}$ of fluid $F_{1}$ is connected. Of course, the boundary conditions force $R_{1}$ to be nonempty. But if there were a second component of $R_{1}$ disjoint from $S_{1}$, then flooding it with $F_{6}$ would reduce cost, since $a_{j 6}<a_{1 j}$. It follows that there is only one connected region of fluid $F_{1}$. Similarly, we see that $R_{2}, R_{3}$ and $R_{4}$ are all connected (by flooding with $F_{6}, F_{5}$ and $F_{5}$, respectively).

Now the following Lemma 4.7 yields a contradiction, as desired.

Lemma 4.7. In the minimizing network $M$ (with both $F_{5}$ and $F_{6}$ present),

- Some component of $R_{5}$ is adjacent to both $R_{2}$ and $R_{4}$.

- Some component of $R_{6}$ is adjacent to both $R_{1}$ and $R_{3}$.

Proof. To begin with, we need a few definitions. For each region $R_{i}$, let $\left|M_{i}\right|=\left|M_{i 5}\right|+\left|M_{i 6}\right|$ denote the total length of the boundary that $R_{i}$ shares with the two additional fluids $F_{5}$ and $F_{6}$. Define the three numerical constants:

$$
\begin{gathered}
C_{1}=g(1,5)=g(3,5)=g(2,6)=g(4,6)=60000, \\
C_{2}=1+\frac{a_{14}+a_{23}-2 a_{45}}{2 a_{35}-a_{13}} \approx 64.07174803043, \text { and } \\
C_{3}=\max \left\{a_{16}-a_{15}, a_{26}-a_{25}, a_{36}-a_{35}, a_{46}-a_{45}\right\} \approx 0.11933797766 .
\end{gathered}
$$

Now, suppose that no component of $R_{5}$ is adjacent to both $R_{2}$ and $R_{4}$. (The other case can be dealt with symmetrically.) First we use flooding to show that $\left|M_{56}\right|$ is relatively small.

By flooding $R_{5}$ with fluid $F_{6}$, we find that

$$
\left|M_{56}\right| \leq C_{3} \frac{\left|M_{1}\right|+\left|M_{2}\right|+\left|M_{3}\right|+\left|M_{4}\right|}{a_{56}} .
$$

By flooding $R_{5} \cup R_{6}$ with $F_{1}$ and then with $F_{3}$, we calculate that

$$
\left|M_{1}\right|+\left|M_{2}\right|+\left|M_{3}\right|+\left|M_{4}\right| \leq C_{2}\left(\left|M_{2}\right|+\left|M_{4}\right|\right) \text {. }
$$


Let $U$ be a connected component of $R_{5}$ that is adjacent to $R_{2}$. By assumption $U$ is not adjacent to $R_{4}$, so by flooding $U$ with $F_{6}$ and using $\left|U_{1}\right|+\left|U_{3}\right|+\left|U_{6}\right| \geq\left|U_{2}\right|$ we find that

$$
\left|U_{1}\right|+\left|U_{3}\right| \geq \frac{a_{56}+a_{25}-a_{26}}{a_{56}+a_{36}-a_{35}}\left|U_{2}\right| \geq \frac{a_{56}-C_{3}}{a_{56}+C_{3}}\left|U_{2}\right|,
$$

where $\left|U_{i}\right|$ denotes the length of the interface $\partial U \cap \partial R_{i}$. Of course, this inequality still holds (trivially) for a connected component $U$ of $R_{5}$ that is not adjacent to $R_{2}$, since then $\left|U_{2}\right|=0$. So by summing over all components of $R_{5}$, we get

$$
\left|M_{25}\right| \leq \frac{a_{56}+C_{3}}{a_{56}-C_{3}}\left(\left|M_{15}\right|+\left|M_{35}\right|\right)
$$

Similarly, we obtain

$$
\left|M_{45}\right| \leq \frac{a_{56}+C_{3}}{a_{56}-C_{3}}\left(\left|M_{15}\right|+\left|M_{35}\right|\right) .
$$

It follows from (8) and (9) that

$$
\left|M_{56}\right| \leq \frac{C_{3}}{a_{56}} C_{2}\left(\left|M_{25}\right|+\left|M_{26}\right|+\left|M_{45}\right|+\left|M_{46}\right|\right) .
$$

Therefore by (10) and (11),

$$
\begin{aligned}
\left|M_{56}\right| & \leq \frac{C_{2} C_{3}}{a_{56}}\left(\left|M_{26}\right|+\left|M_{46}\right|+2 \frac{a_{56}+C_{3}}{a_{56}-C_{3}}\left(\left|M_{15}\right|+\left|M_{35}\right|\right)\right) \\
& \leq \frac{C_{2} C_{3}}{a_{56}} \cdot 2 \frac{a_{56}+C_{3}}{a_{56}-C_{3}}\left(\left|M_{26}\right|+\left|M_{46}\right|+\left|M_{15}\right|+\left|M_{35}\right|\right) \\
& \leq C_{1}\left(\left|M_{26}\right|+\left|M_{46}\right|+\left|M_{15}\right|+\left|M_{35}\right|\right) .
\end{aligned}
$$

Finally, we use a calibration-style calculation to obtain a contradictory estimate that $\left|M_{56}\right|$ is relatively large.

$$
\begin{aligned}
\operatorname{cost}(N) & =\sum_{i<j \leq 4} a_{i j}\left|N_{i j}\right|=\sum_{1 \leq i<j \leq 4} \operatorname{flux}_{N_{i j}}\left(p_{j}-p_{i}\right) \\
& =\sum_{i=1}^{4} \operatorname{flux}_{S_{i}}\left(p_{i}\right)=\sum_{i=1}^{6} \operatorname{flux}_{S_{i}}\left(p_{i}\right) \\
& =\sum_{1 \leq i<j \leq 6} \operatorname{flux}_{M_{i j}}\left(p_{j}-p_{i}\right) \\
& \leq \sum_{1 \leq i<j \leq 6}\left|p_{i}-p_{j}\right| \cdot\left|M_{i j}\right| .
\end{aligned}
$$


Since $M$ was chosen to be cost-minimizing,

$$
\begin{aligned}
0 & >\operatorname{cost}(M)-\operatorname{cost}(N) \\
& \geq \sum_{1 \leq i<j \leq 6} a_{i j}\left|M_{i j}\right|-\sum_{1 \leq i<j \leq 6}\left|p_{i}-p_{j}\right| \cdot\left|M_{i j}\right| \\
& =\sum_{1 \leq i<j \leq 6} g(i, j) \epsilon\left|M_{i j}\right| \\
& =\left(\left|M_{26}\right|+\left|M_{46}\right|+\left|M_{15}\right|+\left|M_{35}\right|\right) C_{1} \epsilon-\left|M_{56}\right| \epsilon,
\end{aligned}
$$

contradicting Eq. (12).

Remark 4.8. If instead we set

$$
g(i, j)= \begin{cases}-1 & \text { if }(i, j)=(5,6) \\ 0 & \text { otherwise }\end{cases}
$$

then $N$ still cannot be calibrated, but $N$ is not minimizing for $F_{1}, F_{2}, \ldots, F_{6}$. So a non-calibrated cone for which placing points for the two absent fluids as close together as possible puts them on opposite vertices of their eyes (i.e., an exception to Theorem 4.4) may or may not be minimizing.

Remark 4.9. Kenneth Brakke ([B1], [B2], [B3]) has considered more general variable-coefficient calibrations than the constant-coefficient calibrations we use. He also considers more general networks (called real flat chains), where fluids are allowed to have fractional densities. We suspect that the network of our counterexample is not minimizing among these realcoefficient networks, and therefore cannot be calibrated even by variablecoefficient flows.

Remark 4.10. For further details on the proof of Theorem 4.6, as well as an alternate counterexample to Conjecture 2.5 whose proof is based on a more complicated calibration-style calculation that keeps track of angles in addition to lengths, see [GNY].

\section{References}

[BTW] Megan Barber, Jennifer Tice and Brian Wecht, Immiscible fluids, Williams College NSF "SMALL" undergraduate research Geometry Group report, 1995.

[B1] Kenneth A. Brakke, Minimal cones on hypercubes, J. Geom. Anal., 1 (1991), 329-338.

[B2] _ Numerical solution of soap film dual problems, Experimental Mathematics, 4 (1995), 269-287.

[B3] _ Soap films and covering spaces, J. Geom. Anal., 5 (1995), 445-514.

[E] Brian Elieson, Cost minimizing networks separating immiscible fluids in $\mathbb{R}^{2}$, Undergraduate thesis, Williams College, 1997. 
[EMN] Alexei Erchak, Ted Melnick and Ramona Nicholson, Double clusters of immiscible fluids, Williams College NSF "SMALL" undergraduate research Geometry Group report, 1996.

[FMMP] David Futer, David McMath, Brian Munson and Sang-Hyoun Pahk, Costminimizing networks among immiscible fluids in $\mathbb{R}^{2}$, Williams College NSF "SMALL" undergraduate research Geometry Group report, 1997.

[GNY] Andrei Gnepp, Ting Ng and Cara Yoder, Two counterexamples on immiscible fluids, Williams College NSF "SMALL" undergraduate research Geometry Group report, 1998.

[HRW] Frank K. Hwang, Dana S. Richards and Pawel Winter, The Steiner tree problem (Annals of Discrete Mathematics 53), North Holland, 1992.

[IT] A.O. Ivanov and A.A. Tuzhilin, Minimal Networks: The Steiner problem and its generalizations, CRC Press, 1994.

[LM] Gary Lawlor and Frank Morgan, Paired calibrations applied to soap films, immiscible fluids, and surfaces or networks minimizing other norms, Pacific J. Math., 166 (1994), 55-83.

[Lay] Steven R. Lay, Convex sets and their applications, Wiley, New York, 1982.

[M1] Frank Morgan, Efficient clusters and networks, SUNY Geneseo Student Workshop organized by Olympia Nicodemi, April 4-5, 1997; participants: Luis Baars, Anna Beckhorn, Wai-Ming Chiu, Brandi Cohey, David Fishman, Sergio Fratarcangelli, Flynn Heiss, Robert Igoe, Travis Jones, Carrie Pitts, Bruce Sutphin, Jeanine Velcenbach, Lisa Wood.

[M2] _ Immiscible fluid clusters in $\mathbb{R}^{2}$ and $\mathbb{R}^{3}$, Michigan Math. J., 45 (1998), 441-450.

[R] R.T. Rockafellar, Convex Analysis, Princeton University Press, 1970.

[W1] Brian White, Existence of least-energy configurations of immiscible fluids, J. Geom. Anal., 6 (1996), 151-161.

[W2] Regularity of the singular sets in immiscible fluid interfaces and solutions to other Plateau-type problems, Proc. Centre Math. Anal. Austral. Nat. Univ., 10 (1985), 244-249.

Received February 12, 1999 and revised August 12, 1999. Research partially supported by an NSF grant.

University of PENN.

E-mail address: dfuter@math.stanford.edu

HARVARD UNIVERSITY

E-mail address: gnepp@post.harvard.edu

StANFORd University

E-mail address: mcmath@math.stanford.edu

UNIVERSITY OF OREGON

E-mail address: munson@math.brown.edu

University of Penn. 
E-mail address: ngtf@seas.upenn.edu

Williams College

E-mail address: spahk@wso.williams.edu

Williams College

E-mail address: cyoder@wso.williams.edu 